Algebraic $\& \mathcal{G}$ Geometric Topology

Volume 2 (2002) 297-309

Published: 24 April 2002

ATG

\title{
Thin position for a connected sum of small knots
}

\author{
YO'AV RIECK \\ ERIC SEDGWICK
}

\begin{abstract}
We show that every thin position for a connected sum of small knots is obtained in an obvious way: place each summand in thin position so that no two summands intersect the same level surface, then connect the lowest minimum of each summand to the highest maximum of the adjacent summand below. See Figure 1.
\end{abstract}

AMS Classification 57M25; 57M27

Keywords 3-manifold, connected sum of knots, thin position

\section{Introduction}

In [3] Gabai introduced thin position, a tool since used for many results on knots and graphs in $S^{3}$ : Gabai's proof of Property $R$ [3], Gordon and Luecke's [4] proof of the Knot Complement Theorem, and Scharlemann and Thompson's [10] proof of Waldhausen's Theorem that irreducible Heegaard splittings of $S^{3}$ are unique, among others. Although thin position has been used in more general settings (eg, by Rieck [8] and Rieck and Sedgwick [9] to study the behavior of Heegaard Surfaces under Dehn surgery) it has been most fruitful when applied to the study of knots in $S^{3}$. We examine thin position in $S^{3}$ for a connected sum of knots.

Given a connected sum of knots, say $K=K_{1} \#$..\# $K_{n}$, there are obvious candidates for thin position of $K$. Choose an ordering of the summands $K_{1}, \ldots, K_{n}$. and put each in a thin position so that successive pairs are separated by level 2 -spheres. Form the connected sum $K$ by connecting the lowest minimum of $K_{i}$ to the highest maximum of $K_{i+1}$. Each level sphere is punctured twice and becomes a decomposing annulus which appears in this presentation as a thin level surface, a surface with a minimum of the knot immediately above and a maximum of the knot immediately below. See Figure 1. We call this position for $K$ a stack on the summands $K_{1}, \ldots, K_{n}$. The width of any stack on these 


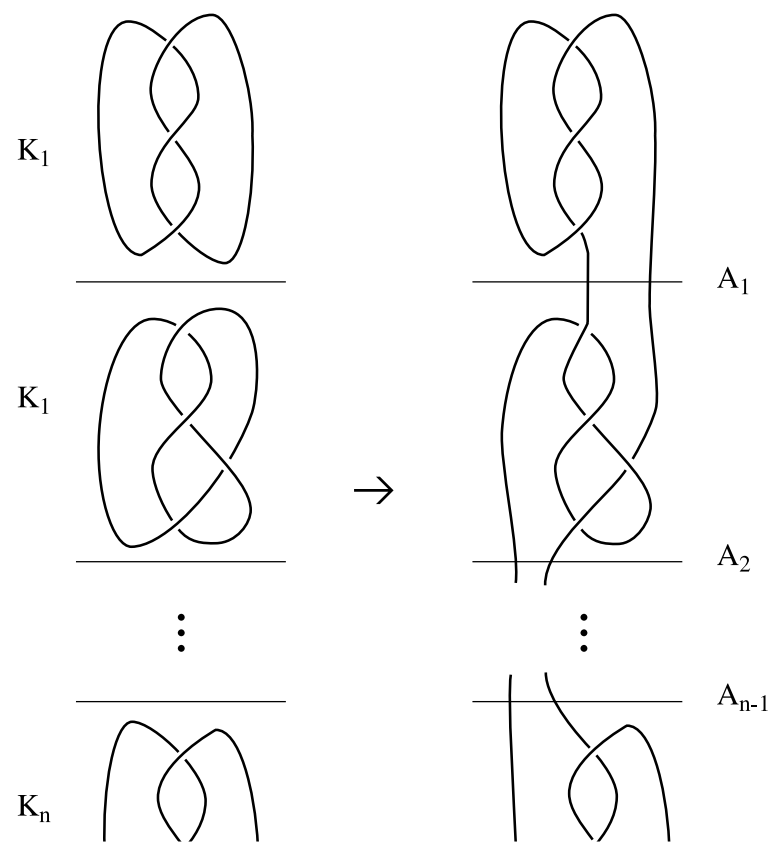

Figure 1: Thin position for a connected sum of small knots is a stack of the summands.

summands is $\sum_{i=1}^{n} w\left(K_{i}\right)-2(n-1)$, which gives an upper bound for the width of $K$.

Is this thin position for $K$ ? In fact, it is not immediately obvious that thin position for a connected sum has any thin levels whatsoever, thin position could be bridge position. While Thompson has shown that small knots do not have thin levels [13], the converse is simply false. In [5] Heath and Kobayashi give an example of a knot (originally considered by Morimoto [6]) for which thin position is bridge position even though its exterior contains an essential four times punctured sphere. Our first theorem gives a converse to Thompson's theorem in the case of a connected sum:

Theorem (4.1) Let $K$ be a connected sum of non-trivial knots, $K=K_{1} \# K_{2}$. Then thin position for $K$ is not bridge position for $K$.

We then analyze thin levels. For $m p$-small knots (inclusive of small knots, see Section 2 for a definition), we demonstrate that each thin level is a decomposing annulus. Indeed, stacks are thin: 
Theorem (5.1) Let $K=\#_{i=1}^{n} K_{i}$ be a connected sum of $m p$-small knots. If $K$ is in thin position, then there is an ordering of the summands $K_{i_{1}}, K_{i_{2}}, \ldots, K_{i_{n}}$ and a collection of leveled decomposing annuli $A_{i_{1}}, A_{i_{2}}, \ldots, A_{i_{n-1}}$ so that the thin levels of the presentation are precisely the annuli $\left\{A_{i_{j}}\right\}$ occurring in order, where the annulus $A_{i_{j}}$ separates the connected sum $K_{i_{1}} \# K_{i_{2}} \# \ldots \# K_{i_{j}}$ from the connected sum $K_{i_{j+1}} \# \ldots \# K_{i_{n}}$. (See Figure 1.)

For $m p$-small knots this yields the equality $w(K)=\sum_{i=1}^{n} w\left(K_{i}\right)-2(n-1)$. Finally, since $m p$-small knots in thin position are also in bridge position [13], each component of the stack is in bridge position. After connecting these knots, the number of maxima is $\sum_{i=1}^{n} b\left(K_{i}\right)-(n-1)$. Thus, while thin position is not bridge position for these knots, it realizes the minimal bridge number as given by Schubert [11] (see Schultens [12] for a modern proof).

Acknowledgements The authors would like to thank Marc Lackenby and Tsuyoshi Kobayashi for helpful conversations, and RIMS of Kyoto University and Nara Women's University for their kind hospitality. The first named author was supported by JSPS grant number P00024.

\section{Preliminaries}

Most of the definitions we use are standard; however we need:

Definition 2.1 A knot $K \subset S^{3}$ is called meridionally planar small if there is no meridional essential planar surface in its complement. We use the notation mp-small.

The set of knots under consideration is substantial and inclusive of small knots: by CGLS [2] small knots in $S^{3}$ are meridionally small, and by definition meridionally small knots are $m p$-small.

The width of a presentation of a link $L$ (ie, the width of an embedding of a compact 1-manifold into $\left.S^{3}\right)$ is defined as follows: let $h: S^{3} \rightarrow[-\infty, \infty]$ be a height function that sends one point to $-\infty$, another to $\infty$ and has all other level sets diffeomorphic to $S^{2}$ (ie, a Morse function with one minimum, one maximum and no other critical points). Suppose $\left.h\right|_{L}$ is Morse (else the width is not defined). Pick a regular value between every two consecutive critical levels of $\left.h\right|_{L}$ and count the number of times $L$ intersects that level. The sum of these numbers is the width of the presentation. Thin position for $L$ is any 
embedding ambient isotopic to $L$ that minimizes the width in that isotopy class. The width of the link $L$, denoted $w(L)$, is the width of a thin position for $L$.

A thin level is a level set of a regular value so that the first critical point of $\left.h\right|_{L}$ above is a minimum and the first critical point below is a maximum. An embedding has no thin levels if and only if each maximum of $L$ occurs above each minimum of $L$. Among all embeddings of $L$ without thin levels, one with a minimal number of maxima (equivalently minima) is called a bridge position for $L$. The bridge number of $L$, denoted $b(L)$, is the number of maxima (equivalently minima) in a bridge position for $L$. By pulling maxima up and minima down, it is easy to see that this is the minimal number of maxima in the isotopy class of the knot.

Given two knots their connected sum is described schematically in Figure 2. For more detail, see [1].

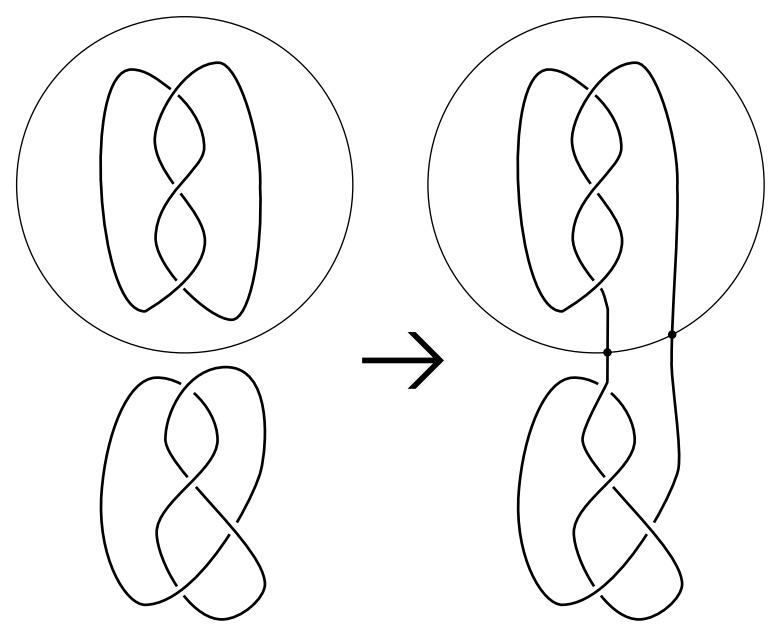

Figure 2: Connected sum of knots.

We recall that every knot has a unique representation as a connected sum of prime knots, ie, any knot $K$ can be written uniquely up to reordering as $\#_{i=1}^{n} K_{i}$ for some $K_{i}$, and $K_{i}$ contains no meridional essential embedded annuli in its exterior. Since any meridional essential embedded annulus decomposes the knot as a connected sum (the factors are not necessarily prime) we call such annulus a decomposing annulus. Note that the uniqueness referred to above is uniqueness of factors, not of the decomposing annuli. A split link on knots $K_{1}$ and $K_{2}$ is an embedding of the two knots that can be separated by an embedded $S^{2}$. 


\section{Essential meridional planar surfaces in a connected sum of $m p$-small knots}

Lemma 3.1 Let $K=\#_{i=1}^{n} K_{i}$ be a connected sum of $m p$-small knots $K_{i}, i=$ $1, \ldots, n$. Then any essential meridional planar surface in the exterior of $K$ is a decomposing annulus.

Proof By way of contradiction, let $n \geq 1$ be the smallest $n$ for which there is a connected sum with $n$ components that is a counterexample to lemma. Let $P$ be a non-annular planar essential surface in the exterior of $K$. By assumption $n>1$, so we can choose an annulus $A$ that decomposes $K$ as a connected sum $K=K_{1} \# K_{2}$. The annulus $A$ is properly embedded in $X=S^{3} \backslash N(K)$ and divides $X$ into manifolds $X_{1}$ and $X_{2}$, the exterior of $K_{1}$ and $K_{2}$ respectively.

Since $A$ and $P$ are essential surfaces in $X, P$ may be isotoped to intersect $A$ essentially. Since both surfaces have meridional boundary, the intersection will be a (perhaps empty) collection of simple closed curves, each curve essential in both surfaces. Among all such positions for $P$, choose one that minimizes the number of curves in the intersection $A \cap P$. Let $P_{i}=P \cap X_{i}, i=1,2$. Each component of the surface $P_{i}$ is properly embedded in the knot exterior $X_{i}$ and is planar. Since $P$ is essential and each curve of $P \cap A$ is essential in $P$, each component of $P_{i}$ is necessarily incompressible in $X_{i}, i=1,2$.

In knot exteriors ( $X_{1}$ and $X_{2}$ in our case) boundary compression implies compression for any surface that is not an annulus. If either $P_{1}$ or $P_{2}$ contains a component that is not an annulus, then that component is also boundary incompressible. This yields an essential surface which is not an annulus, with its boundary on $A$, a meridional slope. Such a component would contradict our assumption that $n$ was the least $n$ so that a connected sum of $n$ components contained an essential planar surface with meridional slope.

We conclude that each $P_{i}$ consists entirely of annuli. This contradicts our assumption that $P$ was a planar surface that is not an annulus.

\section{Thin position is not bridge position}

Theorem 4.1 Let $K$ be a connected sum of non-trivial knots, $K=K_{1} \# K_{2}$. Then any thin position for $K$ is not bridge position for $K$. 
Proof Let $K$ be $K_{1} \# K_{2}$, where $K_{1}$ and $K_{2}$ are not unknots. We will prove the theorem by showing that $K$ has a position that has lower width than its bridge position. Denote the bridge number of $K$ by $b$ and that of $K_{i}$ by $b_{i}(i=1,2)$. By Schubert's Theorem ([11], see [12] for a modern proof) $b=b_{1}+b_{2}-1$.

For a knot in bridge position with $n$ maxima the width of the presentation is easily seen to be

$$
\begin{aligned}
w(K) & =2+4+\ldots+(2 n-2)+2 n+(2 n-2)+\ldots+4+2 \\
& =2\left(\sum_{i=1}^{n} 2 i\right)-2 n \\
& =4 \frac{n(n+1)}{2}-2 n \\
& =2 n^{2} .
\end{aligned}
$$

Applying this to $K$, all we need is to show that $2 b^{2}$ is not the minimal width for $K$.

Consider a presentation of the split link of $K_{1}$ and $K_{2}$ with $K_{1}$ above the level 0 and $K_{2}$ below the level 0 . If we put both knots in bridge position, the width of this presentation is $2 b_{1}^{2}+2 b_{2}^{2}$. We obtain a presentation of $K=K_{1} \# K_{2}$ by connecting the lowest minimum of $K_{1}$ to the highest maximum of $K_{2}$ without introducing new critical points, which lowers the width by 2 . Thus, it suffices to show that $2 b^{2}-\left(2 b_{1}^{2}+2 b_{2}^{2}-2\right)>0$. Applying Schubert's Theorem we get:

$$
\begin{aligned}
2 b^{2}-\left(2 b_{1}^{2}+2 b_{2}^{2}-2\right) & =2\left(b_{1}+b_{2}-1\right)^{2}-\left(2 b_{1}^{2}+2 b_{2}^{2}-2\right) \\
& =\left[2 b_{1}^{2}+2 b_{2}^{2}+4 b_{1} b_{2}-4\left(b_{1}+b_{2}\right)+2\right]-\left[2 b_{1}^{2}+2 b_{2}^{2}-2\right] \\
& =4 b_{1} b_{2}-4\left(b_{1}+b_{2}\right)+4 . \\
& =4\left[b_{1}\left(b_{2}-1\right)-\left(b_{2}-1\right)\right] \\
& =4\left(b_{1}-1\right)\left(b_{2}-1\right) .
\end{aligned}
$$

Since $K_{1}$ and $K_{2}$ are both non-trivial knots, $b_{1}>1$ and $b_{2}>1$ so the above product is positive.

\section{Connected sum of small knots}

Theorem 5.1 Let $K=\#_{i=1}^{n} K_{i}$ be a connected sum of $m p$-small knots. If $K$ is in thin position, then there is an ordering of the summands $K_{i_{1}}, K_{i_{2}}, \ldots, K_{i_{n}}$ and a collection of decomposing annuli $A_{i_{1}}, A_{i_{2}}, \ldots, A_{i_{n-1}}$ so that the thin levels of the presentation are precisely the annuli $\left\{A_{i_{j}}\right\}$ occurring in order, where the annulus $A_{i_{j}}$ separates the connected sum $K_{i_{1}} \# K_{i_{2}} \# \ldots \# K_{i_{j}}$ from the connected sum $K_{i_{j+1}} \# \ldots \# K_{i_{n}}$. (See Figure 1.) 
Proof We induct on $n$. Our goal is to show that there exists a leveled decomposing annulus, ie, an essential annulus that is $h^{-1}(t)$ for some $t$. The theorem will follow since above (and below) the leveled annulus we see summands of $K$ in thin position (else we can reduce the width of $K$ ). Since the prime summands are $m p$-small, Thompson's result [13] guarantees that their thin positions are bridge positions, thus there are no thin level surfaces other than the decomposing annuli.

Existence of a leveled annulus follows in two steps given by the lemmata below.

Lemma 5.2 Let $K$ be the connected sum of $m p$-small knots and $T$ be any thin level in a thin presentation of $K$. Then a neighborhood of a spanning arc of a decomposing annulus is isotopic onto $T$ by an isotopy preserving $K$ setwise.

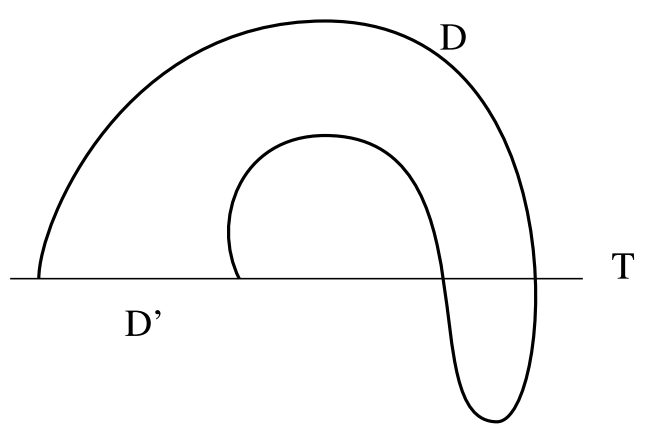

Figure 3: $D^{\prime}$ is incompressible.

The existence of a thin level is guaranteed by Theorem 4.1.

Lemma 5.3 Let $K$ be a connected sum in thin position, and $T$ a thin level onto which a spanning arc of a decomposing annulus is isotopic. Then $T$ is a decomposing annulus.

Remark Lemma 5.3 does not require the assumption that the knots are $m p-$ small. This assumption is only used when, in the proof of Lemma 5.2, we claim that the essential surface we find is (some) decomposing annulus.

We may assume that there is a thin level $T$ that is inessential, for otherwise both lemmata follow from Lemma 3.1. 
Proof of Lemma 5.2 (Cf Thompson [13].) Let $T=h^{-1}\left(t_{0}\right)$ be a thin level. We assumed that $T$ is compressible. Let $D$ be a compressing disk for $T$, and $D^{\prime}$ a punctured disk that $\partial D$ bounds on $T$. Passing to an innermost compression on $D^{\prime}$ we replace $D^{\prime}$ by the innermost disk and accordingly change $D$. (Note: we may not assume that $D$ is a compressing disk for $T$ as it may cross the level $T$ in its interior, see Figure 3 ; we allow $D$ to compress $D^{\prime}$ from above or from below).

We show that $D^{\prime} \cup D$ is essential. If not, it either compresses or boundary compresses. That $D^{\prime} \cup D$ does not compress follows from our innermost choice of $D^{\prime}$ and the fact that the boundary of any compressing disk can be isotoped to be disjoint from $D$. The boundary of any boundary compressing disk that lies in $D^{\prime} \cup D$ can be isotoped to lie entirely in $D^{\prime}$ and is hence a boundary compression for the thin level $T$. A boundary compression that joins a boundary component of $D^{\prime}$ to itself easily implies a compression, which we have already noted does not occur. And, a boundary compression that connects two distinct boundary components can never occur on a thin level. Say that one does, and that it starts above the thin level $T$. By definition of thin level, the first critical point on the knot above $T$ is a minimum. The boundary compressing disk can be used to isotope an arc of the knot to lie below $T$ with just a single maximum. This either pulls a maximum on the arc below the minimum lying above $T$, or eliminates both. Either reduces the width of the presentation. If the arc contains additional critical points of the knot, they are eliminated in pairs, further reducing the width.

Thus, by Theorem 3.1, $D^{\prime} \cup D$ is a decomposing annulus. A spanning arc for this annulus can be isotoped to be disjoint from $D$ and therefore this arc and its neighborhood lie in $D^{\prime}$, hence in $T$.

Proof of Lemma 5.3 This lemma follows from a width calculation. By Lemma 5.2 when we cut $K$ open along a leveled spanning arc on $T$ we get a decomposition of $K$ into $K_{+}$and $K_{-}$, where $K_{+}$and $K_{-}$are non-trivial knots. See Figure 4.

The lemma (and theorem) would follow once we show that $K_{+} \cap T$ and $K_{-} \cap T$ are both empty. Denote the number of times $K_{+}$intersect $T$ by $m_{+}$, and the number of times $K_{-}$intersect $T$ by $m_{-}$. Denoting $m=m_{+}+m_{-}$, our goal is to show that $m=0$. We do this by separating $K_{+}$and $K_{-}$and reconnecting to obtain a new presentation of $K$. See Figure 5 . If $m>0$ we will demonstrate that this manipulation reduces width.

Since $T$ is a thin level for $K$ the first critical point above it is a minimum and the first critical point below it is a maximum. Denote these points by min and 


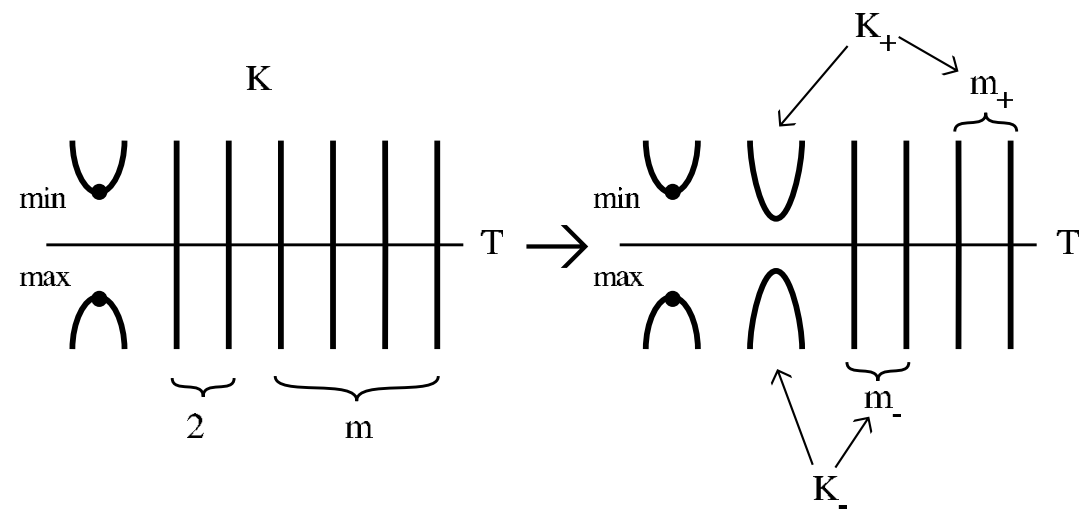

Figure 4: Splitting $K$ to form $K_{+}$and $K_{-}$.
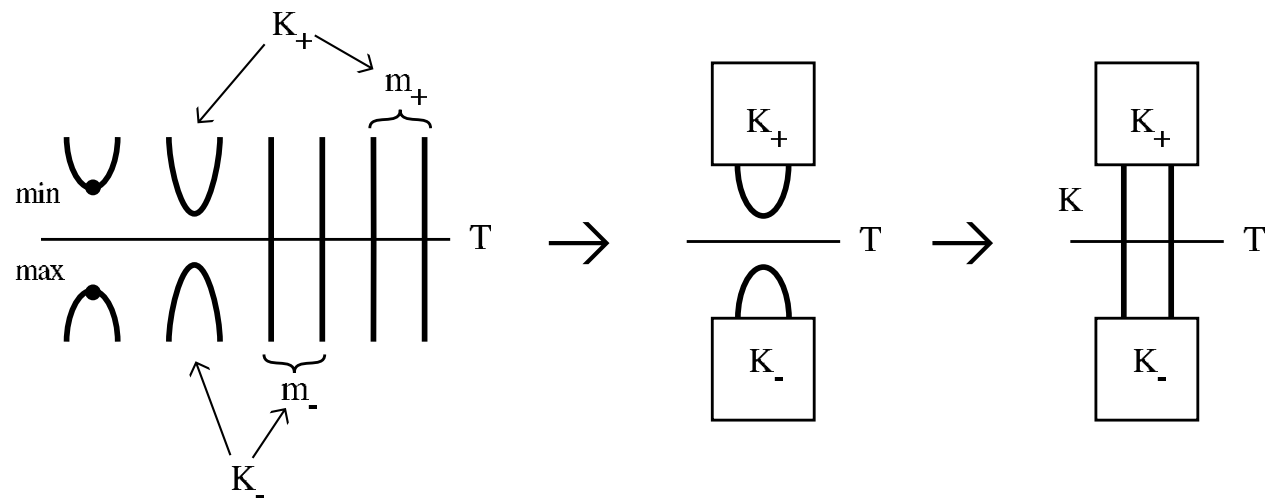

Figure 5: Separating $K_{+}$and $K_{-}$and reconnecting to obtain $K$.

$\max$, see Figure 4. After cutting $K$ open there is another minimum above $T$, which belongs to $K_{+}$, and another maximum below $T$ belonging to $K_{-}$. (We do not know to which of the knots max and min belong.)

We pair each maximum of $K_{+}$with a minimum of $K_{+}$so that for each maximum-minimum pair the minimum is below the maximum. That such correspondence exists follows easily from that fact that above each level there are no less maxima than minima: label the maxima and label the minima, both in descending order, and pair the $i^{\text {th }}$ maximum with the $i^{\text {th }}$ minimum, for example. Similarly we pair up maxima and minima of $K_{-}$.

The following are well known and easy facts about the calculus of width. While isotoping a link, if two maxima change relative heights the width does not change, and similarly for minima. However, if we move a maximum above 
a minimum the width is raised by four, while isotoping a minimum above a maximum the width is lowered by four, see Figure 6 .

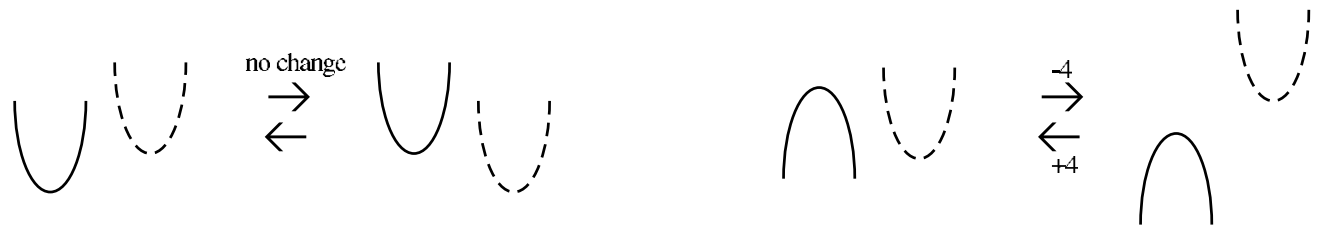

Figure 6: Exchanging relative extrema.

We now begin our width calculation: our first move was cutting $K$ and obtaining the split link on $K_{+}$and $K_{-}$. By doing so, we removed the level $T$ (with $m+2$ punctures) and replaced it by three critical levels, two of width $m+2$ and one of width $m$. Thus we raised the width by $2 m+2$. Next we isotope $K_{+}$ rigidly to lie above $T$ and $K_{-}$to lie below it. See Figure 5 . During this isotopy $K_{+}$and $K_{-}$may intersect each other, but by the end of the process we will once again have the split link on $K_{+}$and $K_{-}$. The isotopy may also temporarily increase the width as a maximum of $K_{+}$is isotoped past a minimum of $K_{-}$; however that contribution will be canceled when the corresponding minimum of $K_{+}$is isotoped past the corresponding maximum of $K_{-}$. Finally, we will connect the lowest minimum of $K_{+}$to the highest maximum of $K_{-}$, obtaining again a presentation of $K$.

For the next definition, see Figure 7.

Definition 5.4 Let $X_{+}, x_{+} Y_{-}, y_{-}$be four critical points so that $X_{+}$(resp. $\left.Y_{-}\right)$is a maximum of $K_{+}$(resp. $\left.K_{-}\right)$and $x_{+}$(resp. $\left.y_{-}\right)$is a minimum of $K_{+}$ (resp. $\left.K_{-}\right)$. Assume further that $X_{+}$is paired with $x_{+}$and $Y_{-}$is paired with $y_{-}$. Then the pair $\left(\left(X_{+}, x_{+}\right),\left(Y_{-}, y_{-}\right)\right)$is called a split pair if $h\left(X_{+}\right)>h\left(y_{-}\right)$ and $h\left(x_{+}\right)<h\left(Y_{-}\right)$.

Split pairs are exactly those pairs which lower the width when we separate $K_{+}$ and $K_{-}$: the maximum $X_{+}$is already higher than the minimum $y_{-}$, so the width is not raised, but the minimum $x_{+}$is below the maximum $Y_{-}$, so the width is lowered by four. In Figure 7 one of the of knots (either dashed or solid) is $K_{+}$and the other is $K_{-}$. Figures (a) and (b) are both split pairs and (c) is not, independent of which knot we choose as $K_{+}$and which $K_{-}$. Being a split pair is a geometric property: both maxima have to be above both minima. Separating a split pair (by moving one knot up and the other down) reduces the width by four regardless of the direction we move the knots. 


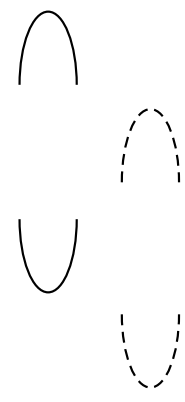

(a)

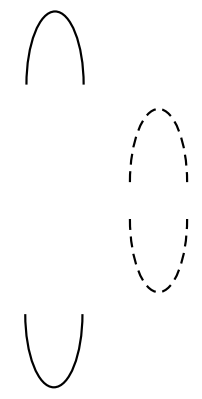

(b)

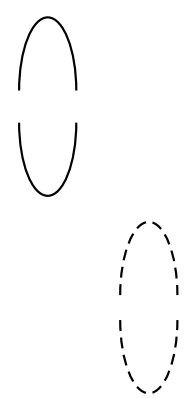

(c)

Figure 7: Two split pairs and a non-split pair.

To get a lower bound on the width reduction we achieve, we must estimate the number of split pairs. There are four possibilities:

(1) $\min \in K_{+}$and $\max \in K_{-}$

(2) $\min \in K_{-}$and $\max \in K_{+}$

(3) $\min \in K_{+}$and $\max \in K_{+}$

(4) $\min \in K_{-}$and $\max \in K_{-}$

$K_{+}$has exactly $\frac{m_{+}}{2}$ maximum-minimum pairs in which the maximum is above the level $T\left(\frac{m_{+}}{2}\right.$ is the number of arcs $K_{+}$has in the ball above $T$ and in the ball below it); similarly $K_{-}$has exactly $\frac{m_{-}}{2}$ pairs separated by $T$. Since all of these minima are below $T$ and the maxima above, we note that the two minima adjacent to $T$ above it and the two maxima below it are not members of these pairs. (We make this comment to ensure that no split pair is counted twice.) With this in mind we are ready to treat each of the cases above:

(1) In this case $K_{+}$has two minima directly above $T$. Each of these minima (together with its corresponding maximum) will be involved in a split pair with each of the pairs of $K_{-}$separated by $T$, a total of $\frac{m_{-}}{2}$ split pairs. Similarly, the two maxima of $K_{-}$below $T$ will be involved in $\frac{m_{+}}{2}$ split pairs each. Since the minima above $T$ and the maxima below it are not members of pairs separated by $T$ no split pair is counted twice. The width is lowered by four per split pair, yielding a reduction of at least $4\left(m_{+}+m_{-}\right)=4 m$.

(2) As in Case (1) the minimum directly above $T$, which is a point of $K_{+}$, is involved in $\frac{m_{-}}{2}$ split pairs. But it is also involved in a split pair with min and its corresponding maximum, and is thus involved in a total of 
$\frac{m_{-}}{2}+1$ split pairs. Similarly, the maximum directly below $T$ (a point of $\left.K_{-}\right)$is involved in $\frac{m_{+}}{2}+1$ split pairs. Each of the $\frac{m_{+}+m_{-}}{2}+2$ split pairs reduces the width by 4 , giving a total of $2 m+8$.

(3) As in Case (1) the minimum directly above $T$ and min are involved in $\frac{m_{-}}{2}$ split pairs each. As in Case (2) the maximum directly below $T$ (a point of $\left.K_{-}\right)$is involved in $\frac{m_{+}}{2}+1$ split pairs. Together we get $m_{-}+\frac{m_{+}}{2}+1$ split pairs, yielding a reduction in width of at least $4 m_{-}+2 m_{+}+4 \geq 2 m+4$.

(4) Symmetric to case (3) we get a reduction of at least $2 m+4$.

Next we reattach the lowest minimum of $K_{+}$to the highest maximum in $K_{-}$to obtain a presentation of $K$. This will reduce width by exactly two. See Figure 5 .

Splitting to form the link increased the width by $2 m+2$ and the final reattachment reduced it by 2 , a net increase of $2 \mathrm{~m}$. In cases 2,3 , and 4 the separation of $K_{+}$and $K_{-}$reduced the width by at least $2 m+8$ (case 2 ) or $2 m+4$ (cases 3 and 4 ). In these cases, we obtain an overall reduction of at least 4 , contradicting thin position. In Case 1 we lowered the width by $4 m$ which yields an overall reduction unless $m=0$, our desired conclusion. (Note that $m=0$ means that $K_{+}$and $K_{-}$do not cross the level $T$ and hence force us to be in case 1.)

This completes the proof of Theorem 5.1.

\section{References}

[1] Gerhard Burde and Heiner Zieschang. Knots. Walter de Gruyter \& Co., Berlin, 1985.

[2] M. Culler, C. McA. Gordon, J. Luecke, and P. B. Shalen. Dehn surgery on knots. Ann. of Math., 125:237-300, 1987.

[3] David Gabai. Foliations and the topology of 3-manifolds. III. J. Differential Geom., 26(3):479-536, 1987.

[4] C. McA. Gordon and J. Luecke. Knots are Determined by Their Complements. J. Amer. Math. Soc., 2:371-415, 1989.

[5] Daniel J. Heath and Tsuyoshi Kobayashi. Essential tangle decomposition from thin position of a link. Pacific J. Math., 179(1):101-117, 1997.

[6] Kanji Morimoto. Tunnel number and connected sum of knots and links. In Proc. Applied Math Workshop, volume 4, pages 117-128, 1994.

[7] Kanji Morimoto. On the super additivity of tunnel number of knots. Math. Ann., 317(3):489-508, 2000. 
[8] Yo'av Rieck. Heegaard structures of manifolds in the Dehn filling space. Topology, 39(3):619-641, 2000.

[9] Yo'av Rieck and Eric Sedgwick. Finiteness results for Heegaard surfaces in surgered manifolds. Comm. Anal. Geom., 9(2):351-367, 2001.

[10] M. Scharlemann and A. Thompson. Thin position and Heegaard splittings of the 3-sphere. J. Diff. Geom., 39(2):343-357, 1994.

[11] Horst Schubert. Über eine numerische Knoteninvariante. Math. Z., 61:245-288, 1954.

[12] Jennifer Schultens. Additivity of bridge number of knots. Preprint, 2001.

[13] Abigail Thompson. Thin position and bridge number for knots in the 3-sphere. Topology, 36(2):505-507, 1997.

Department of Mathematics, Nara Women's University

Kitauoya Nishimachi, Nara 630-8506, Japan, and

Department of Mathematics, University of Arkansas

Fayetteville, AR 72701, USA

and

DePaul University, Department of Computer Science

243 S. Wabash Ave. - Suite 401, Chicago, IL 60604, USA

Email: yoav@mail.uark.edu and esedgwick@cs.depaul.edu

Received: 7 January 2002

Algebraic 83 Geometric Topology, Volume 2 (2002) 